\title{
Of flies and men: insights on organismal metabolism from fruit flies
}

\author{
Akhila Rajan,** and Norbert Perrimon, $1,2 *$
}

\begin{abstract}
The fruit fly Drosophila has contributed significantly to our general understanding of the basic principles of signaling, cell and developmental biology, and neurobiology. However, answers to questions pertaining to energy metabolism have been so far mostly addressed in more complex model organisms such as mice. We review in this article recent studies that show how the genetic tractability and simplicity of Drosophila are being used to identify novel regulatory mechanisms at the organismal level, and to query the co-ordination between energy metabolism and other processes such as neurodegeneration, circadian rhythms, immunity, and tumor biology.
\end{abstract}

Proteins, fats and sugars make up a large proportion of the food we consume. These macromolecules are broken down by evolutionarily conserved biochemical pathways - such as glycolysis, the citric acid cycle and oxidative phosphorylation - to generate the primary energy currency of cells, ATP, and other requisite biomolecules in our body. When nutritional supply exceeds the energy needs, the excess is stored for use at a later time point, such as during starvation, stress or infections. There is a constant flux in the nutritional supply and in the energy needs of an organism. Hence, strategies that maintain a steady state under varying nutritional conditions referred to as energy homeostasis - are crucial for the healthy functioning of an individual. Inability to effectively maintain energy homeostasis results in the development of metabolic disorders like obesity, anorexia and diabetes. Complex metabolic syndromes, with the associated risk of cardiovascular diseases, afflict more than $34 \%$ of the adult population in the United States [1],

*Correspondence: akhila@genetics.med.harvard.edu; perrimon@receptor.med. harvard.edu

'Department of Genetics, Harvard Medical School, 77 Avenue Louis Pasteur, Boston, MA 02115, USA

Full list of author information is available at the end of the article making it expedient to dissect the molecular mechanisms underlying energy homeostasis.

Although Drosophila and humans diverged several hundred million years ago, significant insights have been derived from genetic studies in fruit flies. The genes that determine the body plan and development of fruit flies are evolutionarily conserved and have been found to be crucial also during the early development of human embryos [2,3]. Fundamental components of signaling pathways, including wingless, notch and hedgehog, were originally identified and characterized in fruit flies. Flies are long established as one of the prime models for research in developmental biology, cell biology and neurobiology, but it is only in the past decade that they have been significantly deployed to dissect energy metabolism. This lag is partly attributable to the lack of robust assays for lipid and carbohydrate metabolism and an under-appreciation of the extent to which Drosophila organ systems have functional analogues to vertebrate counterparts. This gap is being filled, however, by a number of recent studies that have led to the characterization of physiological roles for different organs in energy homeostasis in the fly (reviewed in [4-9]).

In this article, we summarize the rationale for using Drosophila as a model for the study of human metabolism, and then discuss in detail a few recent examples that have provided significant and novel insights into organismal metabolism.

\section{Parallels between Drosophila and human physiology}

Many of the organ systems of flies are obviously analogous to those of their vertebrate counterparts: the gut absorbs nutrients, the fat body stores nutrients and functions as a nutrient sensor [10]. The Drosophila heart, a linear tube separated into four compartments by rudimentary valves, is essential for the circulation of nutrients and immune cells; but flies have an open circulatory system, rather than a vascular blood system, and oxygen is delivered by an independent tracheal

\section{Biomed Central}

(C) 2013 Rajan and Perrimon; licensee BioMed Central Ltd. This is an Open Access article distributed under the terms of the Creative Commons Attribution License (http://creativecommons.org/licenses/by/2.0), which permits unrestricted use, distribution, and reproduction in any medium, provided the original work is properly cited. 
system. This uncoupling allows specific aspects of metabolic dysfunction and heart function to be explored without compromising viability. Indeed, the fly has recently emerged as a model for the study of age-related heart dysfunction and polygenic cardiomyopathies (reviewed in $[11,12]$ ), providing insights into high-fatand sugar-induced cardiac dysfunction $[13,14]$.

The insect Malpighian tubules perform basic functions of the kidney such as transport, excretion, and osmoregulation, and fly nephrocytes, a second class of excretory cells present in the body cavity [15], are akin to vertebrate podocytes (cells in the kidney that ultra-filter blood to urine), and are crucial for dealing with metabolic stress and detoxification. It has been argued on the basis of gene expression profiling and the identification of nephrocytes that podocyte biology and renal function can be explored in the fruit fly system [16].

\section{Regulation of metabolism in Drosophila and mammalian systems}

Two key proteins, insulin and adipokinetic hormone (AKH, the fly glucagon) are responsible for regulating carbohydrate and lipid homeostasis. Insulin producing cells (IPCs) in the median neurosecretory (mNSC) region of the fly brain are akin to the pancreatic $\beta$ cells. Corpora cardiaca (CC) cells located in the ring gland (neuroendocrine organ) secrete $\mathrm{AKH}$ and are reminiscent of pancreatic a cells (Figure 1). While insulin promotes the uptake of sugar by tissues from the blood (hemolymph in flies) and its storage as glycogen and fats,
$\mathrm{AKH}$ is secreted during starvation conditions to break down glycogen and fats [17].

Flies express eight Drosophila insulin like peptides (Dilps), which are counterparts of the mammalian insulin and insulin-like growth factors. Among them, Dilp 2, 3 and 5 are expressed in the IPCs [18]. Under conditions of nutrient surplus, Dilps are released from the IPCs [19]. Consistent with their role in maintaining carbohydrate homeostasis, ablation of the IPCs results in elevated circulating sugars [20]. Similar developmental programs are involved in the specification of the IPCs in Drosophila and pancreatic $\beta$ cells in mammals. IPCs develop from a pair of neural stem cells and their differentiation is under the control of the transcription factor Eyeless, whose mammalian homolog, Pax6, is also required for pancreatic beta-cell specification [21]. Deletion of Dilp 1-5 results in phenotypes that model type I diabetes, and include elevated circulating sugar levels, reduced stored fat and initiation of starvation responses, such as autophagy, despite adequate nutrition [22]. Finally, in adult flies, high fat diets (HFDs) and high sugar diets (HSDs) induce obesity and insulin resistance [23-25], suggesting that the molecular mechanisms of insulin resistance are conserved.

The fat body serves as a lipid store similar to the white adipose tissue in mammals. It stores lipids as triacylglycerol (TAG) packed into lipid droplets. A delicate balance between fat storage and breakdown is crucial to maintain energy homeostasis. Under conditions of nutrient deprivation, fat mobilization is triggered by the release from $\mathrm{CC}$ cells of $\mathrm{AKH}$, which activates the

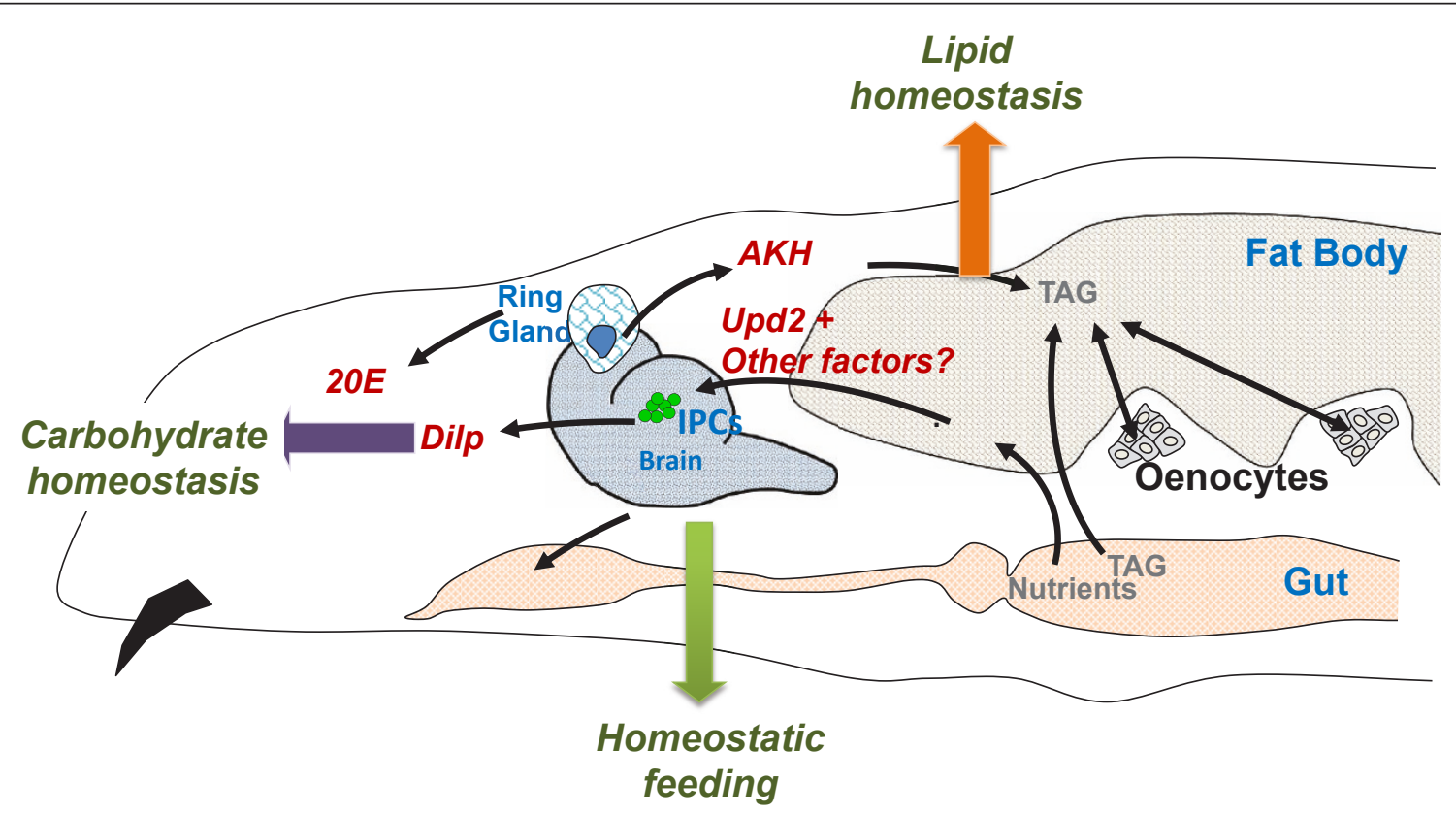

Figure 1. Organs involved in metabolic homeostasis in Drosophila. Hormone names are in red, tissue names in blue, outputs in green. Figure adapted from Leopold and Perrimon [7]. 
G-protein-coupled receptor AKHR to promote lipolysis and glycogen breakdown $[17,26]$. Key components of lipolysis are evolutionarily conserved between mammals and Drosophila, as exemplified by studies on the lipase Brummer (Bmm), the ortholog of the primary mammalian lipase ATGL [27], which is required for basal levels of lipolysis. As with ATGL mutant mice, $B m m$ flies are obese and starvation-sensitive due to their inability to mobilize fat stores during starvation. Another key lipase in mammals is HSL (hormone sensitive lipase), which is required for lipolysis under starvation conditions; its Drosophila homolog plays a similar part in lipid mobilization [28]. The recruitment of lipases to lipid droplets is controlled by perilipin proteins that associate with lipid droplets to promote lipid storage and controlled lipolysis. Two perilipins have been characterized in flies [29]: Lsd-2 promotes fat storage as mutants of Lsd-2 are lean and starvation sensitive [30]; and perilipin-1 mutants display adult onset obesity and hyperphagia [31], suggesting a role for this molecule in controlled access of the lipid droplets to lipolytic enzymes. Together these studies suggest that Drosophila is a suitable system for the study of lipid metabolism.

Until recently, it was thought that fruit flies lacked the crucial human adiposity signal Leptin, believed to be a molecule exclusive to vertebrates. However, a JAK/STAT pathway ligand, Unpaired 2 (Upd2), has been shown to be the functional analogue of human Leptin, with a specific role in the fly fat body in the stimulation of insulin secretion in response to fats in the diet. Remarkably, the nature of the neural circuits through which Upd2 exerts its effects on insulin secretion is very similar to that of the modules on which Leptin acts [32], suggesting that questions pertaining to human Leptin biology can now be addressed in the fly.

In addition to the Leptin-like neuronal circuits, others that sense nutrients are present and have been proposed to be similar to the hypothalamic circuits in humans [33]. For example, a number of neural components that have parallels in mammalian brain, including neuropeptide $\mathrm{F}$ (NPF, ortholog of mammalian neuropeptide Y), serotonergic circuits and hugin neurons that affect feeding, have been discovered in flies [34-36]. Sophisticated behavioral assays have shown how signaling by NPF in olfactory sensory neurons couples hunger state to foraging behavior and how increased insulin signaling under conditions of nutrient surplus inhibits the motivation to feed [37]. Flies also have internal nutrient sensors that regulate food consumption on the basis of its nutritional value [38-40]. Fructosesensing brain neurons have recently been shown to have a receptor called Gr43A that senses levels of fructose in the hemolymph, and controls feeding behavior in a satiation-dependent manner [41].
These examples highlight the parallels between the mammalian and fruit fly systems, and provide compelling evidence for the evolutionary conservation of metabolic regulation. In recent years, studies on fly metabolism have progressed from simply documenting the relevance of Drosophila to human biology, to providing novel insights on mechanisms underlying metabolic regulation. In the sections below, we give a few examples.

\section{New roles for translation inhibitor 4EBP in stress and lifespan extension}

Several studies have established in various model systems (yeast to mice) that nutrient availability alters insulin signaling. The target of rapamycin (TOR) protein kinase is the growth regulatory target of insulin signaling in well fed animals [42,43]. TOR also cell-autonomously responds to amino acids, ATP and oxygen [44]. Thus, TOR signaling connects the intracellular state with the external nutritional status. One of the primary phosphorylation targets of TOR is the translation inhibitor 4EBP [45]. Previous work in mammalian tissue culture assays had implicated 4EBP as a growth regulator, but studies in flies have led to reconsideration of this model, because $4 E B P$ mutant flies show no abnormalities of development or organismal growth, but have severe defects of lipid metabolism [46]. Specifically, 4EBP steady-state mRNA levels are up-regulated under conditions of stress or starvation, and $4 E B P$ mutants exhibit impaired survival under starvation. The starvation sensitivity of the $4 E B P$ mutants has been attributed to impaired fat mobilization, because the mutants burn their fat stores more rapidly than wild-type controls. These findings suggest that $4 \mathrm{EBP}$ acts as a metabolic regulator under stress conditions [47].

Evidence from studies on the extension of lifespan by dietary restriction in flies is also consistent with 4EBP as a metabolic regulator under conditions of stress [47]. Although it is ordinarily an inhibitor of translation, 4EBP can selectively increase translation in stress conditions. Indeed, the translation of genes encoding components of the mitochondrial electron transport chain, and thus mitochondrial activity, were found to be up-regulated by dietary restriction in a 4EBP-dependent fashion. The extension of lifespan by dietary restriction was abolished in $4 E B P$ mutant flies, in which the increase in mitochondrial activity failed to occur. Together, these results suggest that during nutrient stress, a 4EBPmediated metabolic shift toward increased mitochondrial function prolongs lifetime [47].

\section{A neuronal role for S6K in nutritional choice and hunger driven behavior}

The other primary target of TOR is the kinase S6K. In the presence of amino acids, S6K is activated by 
phosphorylation and promotes mRNA translation and growth. Drosophila studies have identified a novel role for S6K in neurons, revealing that it regulates nutrient choice $[48,49]$ and hunger-driven behaviors [50]. All organisms, including fruit flies, choose between different nutritional sources (for example, carbohydrates versus proteins). In nutrient balancing assays - where flies were deprived of either sugars or proteins for three days and then were presented a diet that provided a choice between sugars or protein - flies selectively consumed nutrients that were lacking in their previous diet, thus regaining nutrient homeostasis. These studies also showed that activation of neuronal S6K was crucial for flies to make such a value-based nutritional decision.

\section{Altered lipid metabolism in human neurodegenerative disease models}

Given the energy needs of neuronal cells, it is not surprising that deficits in energy metabolism manifest themselves most prominently in neuronal cell types. Genes that play a role in lipid homeostasis and mitochondrial function have been linked to adult onset neurodegeneration and have been extensively reviewed elsewhere $[4,51,52]$. Here we discuss insights obtained from a fly model of Friedreich's ataxia (FRDA) [53]. FRDA is the most common form of an autosomal recessive neurodegenerative disease affecting the central and peripheral nervous systems. It is caused by reduced expression of the mitochondrial protein frataxin, whose deficiency affects citric acid cycle function. Diabetes is a typical symptom of FRDA patients, and electron microscopic analysis of the neurons and cardiac muscles in mouse models shows an increase in lipid droplets [54], suggesting that there may be changes in lipid metabolism. To pursue further the role played by abnormal lipid metabolism in FRDA pathogenesis, Drosophila frataxin was removed from glial cells (neuronal support cells) by RNA interference (RNAi). This resulted in increased lipid droplet accumulation in glial cells and increased sensitivity to oxidative insults, neurodegeneration and impairment in locomotor activity. Interestingly, overexpression of Glial Lazarillo (GLaz) - the Drosophila homolog of human apolipoprotein $\mathrm{D}$, a carrier protein of lipids - confers a protective effect on the Frataxin-RNA $i$ flies. These studies suggest for the first time a specific requirement for frataxin in glial cells, and open the possibility that the control of lipid metabolism by apolipoproteins could represent a new strategy for the treatment of FRDA patients.

\section{The Warburg effect in Drosophila development}

While the physiology of growth control in insects is very different from that of mammals, a recent study highlights an interesting parallel between metabolic regulation in
Drosophila larval growth and that of tumor growth [55]. Drosophila goes through six different developmental stages - embryonic, three larval, pupal, and adult stimulated by pulses of the steroid hormone ecdysone [56], which provide a recognized system for studying the interplay between metabolism, nutrition and development (see review [57]). The single Drosophila ortholog of the estrogen-related receptor, dERR, has been found to play a key role in carbohydrate and triacylglycerol metabolism. $d E R R$ mutants die as secondinstar larvae with very high circulating sugar levels and very low triacylglycerol levels because of metabolic defects resulting from a decrease in aerobic glycolysis and pentose phosphate shunt. Hence, $d E R R$ promotes a proliferative metabolic program favoring the conversion of carbohydrates to biomass during larval development. This is reminiscent of the metabolic state of cancer cells, which use aerobic glycolysis to convert nutrients into biomass as opposed to energy, a metabolic adaptation to sustained growth known as the Warburg effect [58]. The authors of the paper point out that the three mammalian ERRs are associated with cancer progression, suggesting an ancestral framework for the association between ERRs and rapid growth.

\section{High-throughput screens to identify novel players in lipid metabolism}

The power of the Drosophila system is the opportunity it offers to use high-throughput screens with robust assays to identify novel players in fundamental processes. This is well illustrated by recent genome-wide screens in both flies and in Drosophila S2 cells. For example, a genomewide transgenic RNAi screen in adult flies for altered triglyceride levels identified a role for hedgehog signaling in adipocyte determination [2]. The screen identified a large number of genes already known to play a key part in mammalian fat or lipid metabolism, as well as a plethora of candidate genes for a role in the regulation of triacylglycerol storage, a large proportion of which had no previous annotated biological function. Another study [59] used buoyancy-based screen of Drosophila larvae to identify fat-storage mutants. Finally, a screen for regulators of lipid droplet formation and utilization in Drosophila S2 cells was the first to identify a requirement for functional Golgi vesicular transport in lipolysis [60]. The tools available in Drosophila to allow effective genetic screens are likely continue to identify novel regulatory mechanisms to inform investigations in mammalian systems.

In addition to the examples cited above, studies in Drosophila have identified links between the complex processes of immunity and circadian rhythms and that of organismal metabolism (reviewed in [5]). For example, the gut microbiota was recently found to influence host 
metabolism by impinging on TOR signaling [61]. Similarly, regulation of circadian factors by posttranslational sugar modifications in response to nutrient status has been observed in fruit flies and found to be conserved in mice $[62,63]$. In the future, we can expect more contributions from such studies in Drosophila to inform us of how inter-organ communication signals contribute to organismal metabolic homeostasis.

This article is part of the BMC Biology tenth anniversary series. Other articles in this series can be found at http://www.biomedcentral.com/ bmcbiol/series/tenthanniversary.

\section{Author details}

'Department of Genetics, Harvard Medical School, 77 Avenue Louis Pasteur, Boston, MA 02115, USA. ${ }^{2}$ Howard Hughes Medical Institute, 77 Avenue Louis Pasteur, Boston, MA 02115, USA

\section{Published: 15 April 2013}

\section{References}

1. Ervin RB: Prevalence of metabolic syndrome among adults 20 years of age and over, by sex, age, race and ethnicity, and body mass index: United States, 2003-2006. Natl Health Stat Rep 2009, 13:1-8.

2. Nusslein-Volhard C, Wieschaus E: Mutations affecting segment number and polarity in Drosophila. Nature 1980, 287:795-801.

3. Nickel W, Seedorf M: Unconventional mechanisms of protein transport to the cell surface of eukaryotic cells. Annu Rev Cell Dev Bio/ 2008, 24:287-308.

4. Baker KD, Thummel CS: Diabetic larvae and obese flies-emerging studies of metabolism in Drosophila. Cell Metab 2007, 6:257-266.

5. Rajan A, Perrimon N: Drosophila as a model for interorgan communication: lessons from studies on energy homeostasis. Dev Cell 2011, 21:29-31.

6. Melcher C, Bader R, Pankratz MJ: Amino acids, taste circuits, and feeding behavior in Drosophila: towards understanding the psychology of feeding in flies and man. J Endocrinol 2007, 192:467-472.

7. Leopold P, Perrimon N: Drosophila and the genetics of the internal milieu. Nature 2007, 450:186-188.

8. Kühnlein RP: Drosophila as a lipotoxicity model organism--more than a promise? Biochim Biophys Acta 2010, 1801:215-221.

9. Bharucha KN: The epicurean fly: using Drosophila melanogaster to study metabolism. Pediatr Res 2009, 65:132-137.

10. Colombani J, Raisin S, Pantalacci S, Radimerski T, Montagne J, Leopold P: A nutrient sensor mechanism controls Drosophila growth. Cell 2003, 114:739-749.

11. Nishimura M, Ocorr K, Bodmer R, Cartry J: Drosophila as a model to study cardiac aging. Exp Gerontol 2011, 46:326-330.

12. Qian $L$, Bodmer R: Probing the polygenic basis of cardiomyopathies in Drosophila. J Cell Mol Med 2012, 16:972-977.

13. Birse RT, Choi J, Reardon K, Rodriguez J, Graham S, Diop S, Ocorr K, Bodmer R, Oldham S: High-fat-diet-induced obesity and heart dysfunction are regulated by the TOR pathway in Drosophila. Cell Metab 2010, 12:533-544.

14. Na J, Musselman LP, Pendse J, Baranski TJ, Bodmer R, Ocorr K, Cagan R: A Drosophila model of high sugar diet-induced cardiomyopathy. PLOS Genet 2013, 9:e1003175

15. Andrei C, Dazzi C, Lotti L, Torrisi MR, Chimini G, Rubartelli A: The secretory route of the leaderless protein interleukin 1 beta involves exocytosis of endolysosome-related vesicles. Mol Biol Cell 1999, 10:1463-1475.

16. MacKenzie A, Wilson HL, Kiss-Toth E, Dower SK, North RA, Surprenant A: Rapid secretion of interleukin-1 beta by microvesicle shedding. Immunity 2001, 15:825-835

17. Kim SK, Rulifson EJ: Conserved mechanisms of glucose sensing and regulation by Drosophila corpora cardiaca cells. Nature 2004, 431:316-320.

18. Gronke S, Clarke DF, Broughton S, Andrews TD, Partridge L: Molecular evolution and functional characterization of Drosophila insulin-like peptides. PLoS Genet 2010, 6:e1000857.

19. Geminard C, Rulifson EJ, Leopold P: Remote control of insulin secretion by fat cells in Drosophila. Cell Metab 2009, 10:199-207.
20. Rulifson EJ, Kim SK, Nusse R: Ablation of insulin-producing neurons in flies: growth and diabetic phenotypes. Science 2002, 296:1118-1120.

21. Wang S, Tulina N, Carlin DL, Rulifson EJ: The origin of islet-like cells in Drosophila identifies parallels to the vertebrate endocrine axis. Proc Natl Acad SciU S A 2007, 104:19873-19878.

22. Zhang H, Liu J, Li CR, Momen B, Kohanski RA, Pick L: Deletion of Drosophila insulin-like peptides causes growth defects and metabolic abnormalities. Proc Natl Acad Sci U S A 2009, 106:19617-19622.

23. Musselman LP, Fink JL, Narzinski K, Ramachandran PV, Hathiramani SS, Cagan $\mathrm{RL}$, Baranski TJ: A high-sugar diet produces obesity and insulin resistance in wild-type Drosophila. Dis Model Mech 2011, 4:842-849.

24. Pasco MY, Léopold P: High sugar-induced insulin resistance in Drosophila relies on the lipocalin neural lazarillo. PloS One 2012, 7:e36583.

25. Hull-thompson J, Muffat J, Sanchez D, Walker DW, Benzer S, Ganfornina MD Jasper $\mathrm{H}$ : Control of metabolic homeostasis by stress signaling is mediated by the lipocalin NLaz. PLoS Genet 2009, 5:e1000460.

26. Lee G, Park JH: Hemolymph sugar homeostasis and starvation-induced hyperactivity affected by genetic manipulations of the adipokinetic hormone-encoding gene in Drosophila melanogaster. Genetics 2004 167:311-323.

27. Gronke S, Mildner A, Fellert S, Tennagels N, Petry S, Muller G, Jackle H, Kuhnlein RP: Brummer lipase is an evolutionary conserved fat storage regulator in Drosophila. Cell Metab 2005, 1:323-330.

28. Bi J, Xiang Y, Chen H, Liu Z, Gronke S, Kuhnlein RP, Huang X: Opposite and redundant roles of the two Drosophila perilipins in lipid mobilization. $J$ Cell Sci 2012, 125:3568-3577.

29. Miura S, Gan JW, Brzostowski J, Parisi MJ, Schultz CJ, Londos C, Oliver B, Kimmel AR: Functional conservation for lipid storage droplet association among Perilipin, ADRP, and TIP47 (PAT)-related proteins in mammals, Drosophila, and Dictyostelium. J Bio/ Chem 2002, 277:32253-32257.

30. Gronke S, Beller M, Fellert S, Ramakrishnan H, Jackle H, Kuhnlein RP: Control of fat storage by a Drosophila PAT domain protein. Curr Bio/ 2003, 13:603-606

31. Beller M, Bulankina AV, Hsiao H-H, Urlaub H, Jäckle H, Kühnlein RP: PERILIPINdependent control of lipid droplet structure and fat storage in Drosophila. Cell Metab 2010, 12:521-532.

32. Rajan A, Perrimon N: Drosophila cytokine Unpaired 2 regulates physiological homeostasis by remotely controlling insulin secretion. Cell 2012, 151:123-137.

33. Al-Anzi B, Sapin V, Waters C, Zinn K, Wyman RJ, Benzer S: Obesity-blocking neurons in Drosophila. Neuron 2009, 63:329-341.

34. Wu Q, Wen T, Lee G, Park JH, Cai HN, Shen P: Developmental control of foraging and social behavior by the Drosophila neuropeptide Y-like system. Neuron 2003, 39:147-161.

35. Kaplan DD, Zimmermann G, Suyama K, Meyer T, Scott MP: A nucleostemin family GTPase, NS3, acts in serotonergic neurons to regulate insulin signaling and control body size. Genes Dev 2008, 22:1877-1893.

36. Melcher C, Pankratz MJ: Candidate gustatory interneurons modulating feeding behavior in the Drosophila brain. PLOS Biol 2005, 3:e305.

37. Root CM, Ko Kl, Jafari A, Wang JW: Presynaptic facilitation by neuropeptide signaling mediates odor-driven food search. Cell 2011, 145:133-144.

38. Burke CJ, Waddell S: Remembering nutrient quality of sugar in Drosophila. Curr Biol 2011, 21:746-750.

39. Dus M, Min S, Keene AC, Lee GY, Suh GS: Taste-independent detection of the caloric content of sugar in Drosophila. Proc Natl Acad Sci U S A 2011, 108:11644-11649.

40. Fujita M, Tanimura T: Drosophila evaluates and learns the nutritional value of sugars. Curr Biol 2011, 21:751-755

41. Miyamoto T, Slone J, Song $\mathrm{X}$, Amrein $\mathrm{H}$ : A fructose receptor functions as a nutrient sensor in the Drosophila brain. Cell 2012, 151:1113-1125

42. Zhang H, Stallock JP, Ng JC, Reinhard C, Neufeld TP: Regulation of cellular growth by the Drosophila target of rapamycin dTOR. Genes Dev 2000, 14:2712-2724

43. Oldham S, Montagne J, Radimerski T, Thomas G, Hafen E: Genetic and biochemical characterization of dTOR, the Drosophila homolog of the target of rapamycin. Genes Dev 2000, 14:2689-2694.

44. Wullschleger $\mathrm{S}$, Loewith $\mathrm{R}$, Hall MN: TOR signaling in growth and metabolism. Cell 2006, 124:471-484

45. Hay N, Sonenberg N: Upstream and downstream of mTOR. Genes Dev 2004 18:1926-1945.

46. Teleman AA, Chen YW, Cohen SM: 4E-BP functions as a metabolic brake 
used under stress conditions but not during normal growth. Genes Dev 2005, 19:1844-1848.

47. Zid BM, Rogers AN, Katewa SD, Vargas MA, Kolipinski MC, LuTA, Benzer S, Kapahi P: 4E-BP extends lifespan upon dietary restriction by enhancing mitochondrial activity in Drosophila. Cell 2009, 139:149-160.

48. Ribeiro C, Dickson BJ: Sex peptide receptor and neuronal TOR/S6K signaling modulate nutrient balancing in Drosophila. Curr Bio/ 2010, 20:1000-1005.

49. Vargas MA, Luo N, Yamaguchi A, Kapahi P: A role for $\mathrm{S} 6$ kinase and serotonin in postmating dietary switch and balance of nutrients in $D$. melanogaster. Curr Biol 2010, 20:1006-1011.

50. Wu Q, Zhang Y, Xu J, Shen P: Regulation of hunger-driven behaviors by neural ribosomal S6 kinase in Drosophila. Proc Natl Acad Sci U S A 2005, 102:13289-13294

51. Lessing $D$, Bonini NM: Maintaining the brain: insight into human neurodegeneration from Drosophila melanogaster mutants. Nat Rev Genet 2009, 10:359-370

52. Ioannou YA: Niemann-Pick C proteins in sterol transport and absorption: flies in the ointment. Dev Cell 2007, 12:481-483.

53. Navarro JA, Ohmann E, Sanchez D, Botella JA, Liebisch G, Molto MD, Ganfornina MD, Schmitz G, Schneuwly S: Altered lipid metabolism in a Drosophila model of Friedreich's ataxia. Hum Mol Genet 2010, 19:2828-2840.

54. Puccio H, Simon D, Cossee M, Criqui-Filipe P, Tiziano F, Melki J, Hindelang C, Matyas R, Rustin P, Koenig M: Mouse models for Friedreich ataxia exhibit cardiomyopathy, sensory nerve defect and Fe-S enzyme deficiency followed by intramitochondrial iron deposits. Nat Genet 2001, 27:181-186

55. Tennessen JM, Baker KD, Lam G, Evans J, Thummel CS: The Drosophila estrogen-related receptor directs a metabolic switch that supports developmental growth. Cell Metab 2011, 13:139-148.
56. Thummel CS: Flies on steroids--Drosophila metamorphosis and the mechanisms of steroid hormone action. Trends Genet 1996, 12:306-310.

57. Edgar BA: How flies get their size: genetics meets physiology. Nat Rev Genet 2006, 7:907-916

58. Vander Heiden MG, Cantley LC, Thompson CB: Understanding the Warburg effect: the metabolic requirements of cell proliferation. Science 2009, 324:1029-1033.

59. Reis T, Van Gilst MR, Hariharan IK: A buoyancy-based screen of Drosophila larvae for fat-storage mutants reveals a role for Sir 2 in coupling fat storage to nutrient availability. PLoS Genet 2010, 6:e1001206.

60. Guo Y, Walther TC, Rao M, Stuurman N, Goshima G, Terayama K, Wong JS, Vale $\mathrm{RD}$, Walter $\mathrm{P}$, Farese RV: Functional genomic screen reveals genes involved in lipid-droplet formation and utilization. Nature 2008, 453:657-661.

61. Storelli G, Defaye A, Erkosar B, Hols P, Royet J, Leulier F: Lactobacillus plantarum promotes Drosophila systemic growth by modulating hormonal signals through TOR-dependent nutrient sensing. Cell Metab 2011, 14:403-414.

62. Kim EY, Jeong EH, Park S, Jeong HJ, Edery I, Cho JW: A role for OGlcNAcylation in setting circadian clock speed. Genes Dev 2012, 26:490-502.

63. Kaasik K, Kivimae S, Allen JJ, Chalkley RJ, Huang Y, Baer K, Kissel H, Burlingame AL, Shokat KM, Ptacek LJ et al: Glucose sensor O-GIcNAcylation coordinates with phosphorylation to regulate circadian clock. Cell Metab 2013, 17:291-302.

doi:10.1186/1741-7007-11-38

Cite this article as: Rajan A, Perrimon N: Of flies and men: insights on organismal metabolism from fruit flies. BMC Biology 2013, 11:38. 